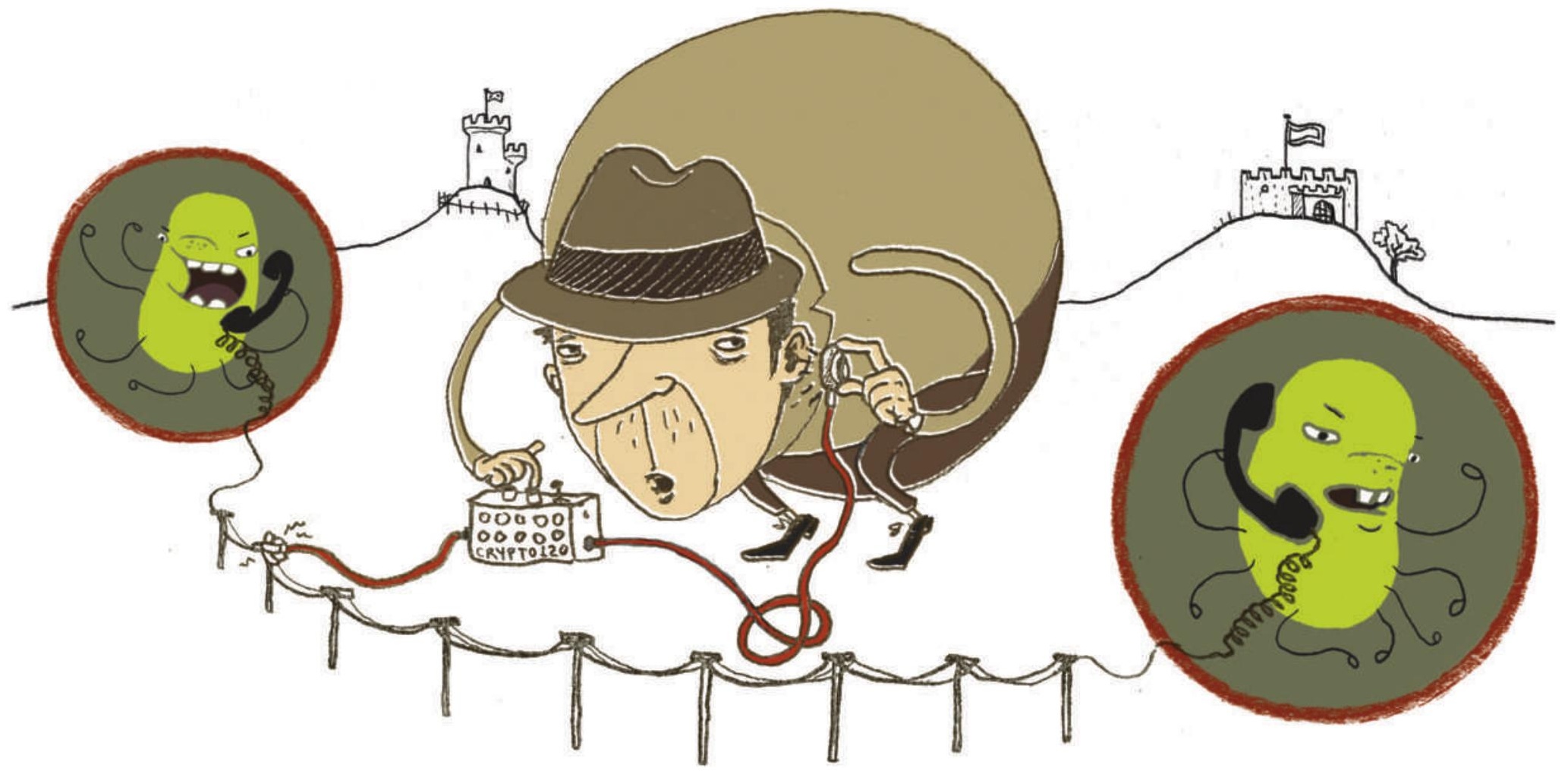

\title{
TINKER, BACTERIA, EUKARYOTE, SPY
}

\section{Bacteria and their hosts may reside in different kingdoms, but that doesn't stop them from intercepting each other's communications. Asher Mullard reports.}

W hen Mark Lyte looked up from the podium at the 1992 American Society for Microbiology meeting in New Orleans, Louisiana, he saw two faces - and nearly 400 empty seats. Lyte, a microbiologist at Texas Tech University in Lubbock, ploughed on regardless. He had a lot to say. His experiments had shown that three species of infectious bacterium intercept the human stress-response hormone noradrenaline and use it as a cue to escalate their growth - perhaps explaining why stressed animals are more likely to die of infection despite having boosted their immune responses.

Minutes into Lyte's lecture, one person got up and walked down the long, lonely aisle to the door. Only his loyal technician remained, along with the two people chairing the session. After the thin applause, one of them posed a question: "Why would you ever want to do these experiments?" Researchers already knew that bacteria and humans detected each other's presence through membrane receptors and cell-wall molecules - but no one thought that bacteria were sophisticated enough to eavesdrop on the long-range chemical signals of the organisms that host them.

Nowadays, Lyte's lectures are packed, and a burgeoning field of researchers is studying the chemical crosstalk between bacteria and their hosts. They know that, as Lyte showed, bacteria respond to the chemical signals that their hosts use for internal communications; they've also discovered that infected hosts intercept the signals that bacteria send to each other, apparently to confound their knavish tricks. The bacteria fight back, turning off immune responses. Interkingdom espionage offers all the intrigue, jamming, fakery and subversion that you could find in a good spy thriller.

Some scientists see all this subversion as something to emulate: if body cells can confuse bacterial attackers with this sort of crosstalk, why shouldn't pharmaceutical companies? Hence the search for small molecules or antibodies that could serve as new classes of antibacterials. And on top of - or beneath - these practical opportunities, there are also deeper scientific questions. How did organisms as different as bacteria and their eukaryotic hosts come to understand each other in the first place? And is it possible that the crosstalk underlies mechanisms of cooperation as well as conflict?

For centuries, bacteria were thought to be loners that didn't communicate with one another, let alone with anything else. Bonnie Bassler, who studies bacterial communication at Princeton University in New Jersey, recalls a time when many of her colleagues thought that "bacteria didn't have the genetic power to do anything interesting - they ate, they moved, they divided". But in the 1970s, researchers discovered that Vibrio fischeri, bacteria that live in squid, fish and the open ocean, coordinate their bioluminescence by sensing the level of signalling molecules given off by others ${ }^{1}$, a system that later came to be called quorum sensing. Signalling that synchronizes bacterial gene expression patterns and coordinates behaviour within a population has now been seen in all sorts of bacteria - and it is used for various purposes, including establishing infection and increasing virulence.

\section{Over the wall}

Still fighting against the idea that bacteria were simpletons, Lyte's study ${ }^{2}$ in 1992 was one of the first to show that bacteria also detect signalling molecules released by the organisms that they infect. In 2006, microbiologist Vanessa Sperandio, at the University of Texas Southwestern Medical Center at Dallas, and her colleagues showed how intimately the two communication systems could be integrated. Sperandio's team found that QseC, a bacterial receptor that detects a quorum-sensing signal called autoinducer 3 (AI-3), is also activated by the mammalian hormones adrenaline and noradrenaline ${ }^{3}$. Both cause the bacterium Escherichia coli to express virulence genes. Sperandio suspects that AI-3 and the human hormones have structural similarities that enable them to bind to 
the QseC receptor, and is looking into whether human hormone receptors can also detect AI-3.

Some argue that this crosstalk is not 'signalling' at all - it did not evolve specifically as a means for two willing parties to communicate. But the fact that the same receptor performs this double duty still requires explanation. Some invoke convergent evolution, suggesting that functional requirements led bacteria and their hosts to evolve chemical messengers with some similar characteristics. An alternative possibility - a controversial one, but one to which Sperandio subscribes - is that the same receptor works for both bacterial and eukaryotic signals because eukaryotic cells acquired the genes for cellular communication

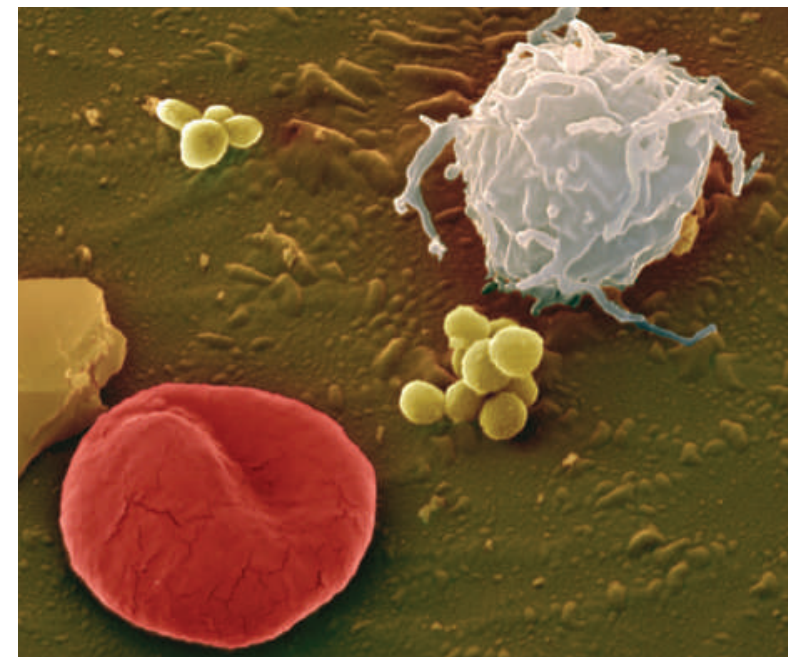

Who's listening in? Staphylococcus bacteria between two body cells.

bacteria in check," says Gresham. She $\overrightarrow{0}$ suspected that some component of human blood plasma was interfering 풌 with $S$. aureus communication.

In 2008, after painstakingly screening serum samples, Gresham and her team found that component: apolipoprotein $\mathrm{B}$ (APOB), a huge lipidbinding protein that helps transport cholesterol in the bloodstream ${ }^{7}$. Gresham found that APOB smothers an $S$. aureus quorum-sensing molecule called autoinducing peptide 1 (AIP1), cutting the line of communication used to coordinate the onset of virulence. Mice chemically or genetically manipulated to lack APOB are more susceptible to MRSA.

In this case at least, says Gresham, both sides benefit: the host prevents

"C12 acts as a stealth agent," Kaufman says. "P. aeruginosa might use it to shut down the immunity locally so that by the time the host realizes there is something there, it is too late," he says.

Dirty play goes both ways. Plants and algae, for example, are master mimics of bacterial quorum-sensing signals. One of the bestknown examples is found in the red alga Delisea pulchra, which produces quorum-sensing signal lookalikes called furanones. In 2002, microbiologist Staffan Kjelleberg of the University of New South Wales in Sydney, Australia, and his colleagues showed that furanones jam signalling in P. aeruginosa and in E. coli, probably either by competing with native quorum-sensing signals or by changing the configuration hijacking," says Kendra Rumbaugh, who studies interkingdom signalling at Texas Tech University.

Take the microbial messenger C12. This is a quorum-sensing signal that coordinates the expression of virulence genes for Pseudomonas aeruginosa, a pathogen that can infect burn injuries or people with supressed immune systems. When Gunnar Kaufman from the Scripps Research Institute in La Jolla, California, started working on it in 2005 he knew it was detected by mammals, and several studies suggested that it helped to trigger inflammation. "But it's the opposite," Kaufman says.

When he and his colleagues treated mice with $\mathrm{C} 12$ they found that it actually inhibits the NF-kB signalling pathway ${ }^{5}$, which is crucial for immune response; studies on human cells indicated much the same. While it might make sense for the host immune system to listen out for the quorum-sensing signal, in this case the bacteria seem to have evolved the upper hand. an infection from turning pathogenic, and the bacteria are able to live happily in the nose without threat from the host's immune system. Through mutual surveillance and manipulation, the host and the pathogen can "arrive at a détente”, says Gresham. If the balance breaks down, because a patient is old, sick or otherwise immunocompromised, then the infection starts escalating out of control. Clinical studies have shown that APOB levels are lower in critically ill patients than in healthy individuals, which Gresham thinks could partly explain why these patients are highly vulnerable to MRSA infection. "Therapeutically, is there a way to lower that risk by giving these patients APOB, or a peptide mimetic of APOB?" she wonders.

Researchers have been trying to manipulate quorum sensing to make antibacterial drugs since the 1990s. They have had little success; a fair few early start-ups based on the idea died. One problem may have been the dearth of knowledge about interactions between bacterial signals and host signals. "It's hard to think about developing a drug that targets quorum sensing without knowing how the host deals with [quorum sensing]," says Gresham. Researchers are wary of blocking a quorum-sensing signal if the host might already be using it to gauge its immune response, or of developing a compound that risks inhibiting both bacterial and host receptors.

Still, many microbiologists and chemists are still hopeful that they can design neat little molecules to artificially stifle or manipulate microbial communication systems more effectively than the systems that have evolved naturally. "This field is kind of like a sandbox for chemists," says Helen Blackwell, a chemist from the University of Wisconsin, Madison. "If we can understand these signals better, and learn what components of these signals 
are necessary at the molecular level, then we can tinker with them and start to engage the bacteria in new conversations, and we can try to confuse them."

Working in close collaboration with chemists, pharmacologists and other microbiologists, Sperandio screened 150,000 molecules for inhibitors of the quorum-sensing receptor QseC and identified one, LED209, as a potent, relatively non-toxic small molecule that protects mice from both Salmonella typhimurium and Francisella tularensis, although not from pathogenic E. coli ${ }^{8}$. In 2008, the group won US\$6.5 million over 5 years from the US National Institutes of Health to search for LED209 analogues that provide greater protection and lower toxicity. These, they hope, will find use as broad-spectrum therapeutics to protect patients from stubborn infections associated with assisted-breathing apparatus, several of which have receptors much like QseC. "Our idea is to have this in a preclinical form in 5 years," she says.

For Nafsika Georgopapadakou, though, the anti-quorum-sensing approach is ultimately flawed. Georgopapadakou, a consultant in Montreal, Canada, has worked on antimicrobials at several large companies. She says that quorum sensing seems to be important for establishing infections rather than for maintaining them, and so such therapeutics are only likely to be useful as prophylactics that are given before the infection has started. Anti-quorum-sensing approaches don't kill bacteria, she adds, they just lower virulence and increase the odds that antibiotics and the immune system can clear the infection. "If I'm going to sweat making novel

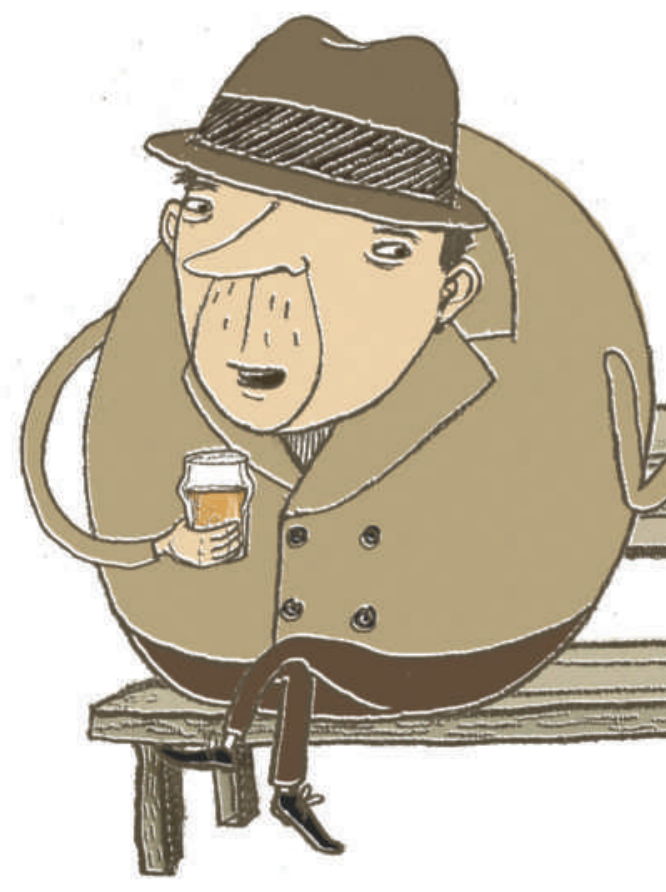

compounds, I would much rather kill bugs."

But proponents of anti-quorum-sensing approaches argue that their non-lethal approach is actually advantageous. One of the main failings of current antibiotics is that their efficient killing drives the rapid evolution of drug resistance, says Sperandio. Anti-quorum-sensing strategies, by contrast, could have a much longer shelf life. "If you don't kill the bacteria, you're not speeding up the process of

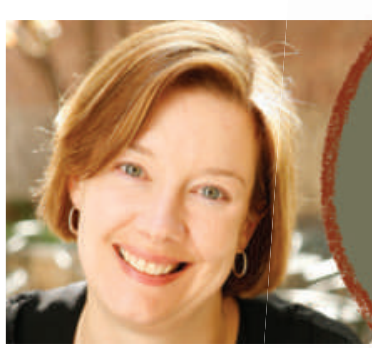

developing resistance that much," she says. To get the full effect, however, they will probably have to be used with other drugs, she adds.

\section{The spy who loved me}

Some researchers are less interested in intervening in bacteria-host communication and more interested in exploring why it happens. "Everybody, including our lab, has focused on pathogenic processes," says Rumbaugh. "Unfortunately, the field might be focusing on the wrong direction." As Rumbaugh sees it, "pathogenesis is the exception". Many microbiologists believe that these crosskingdom communication systems evolved because they served a beneficial purpose for both sides, by supporting mutually beneficial relationships between bacteria and hosts. "So, what are the real functions of these [interkingdom exchanges]?" Rumbaugh asks.

Both Sperandio and Rumbaugh suspect that there is a host of as-yet-unidentified small molecules that pass between bacteria and humans, and that these need to be isolated and catalogued if researchers are going to understand the full scope of interkingdom communication and the purposes that it serves. Practically, this could be tough. Quorum-sensing signals can be hard to distinguish from other soluble chemicals; some of them are only produced in specific environmental conditions that can be near-impossible to reproduce in a culture dish, and some are manufactured in such minute quantities that they are difficult to collect and analyse. The structure of AI-3, for instance, has not yet been resolved for this reason, Sperandio says.

There is an additional obstacle to deciphering friendly bacterial-host communication. Just as vice and espionage tend to capture the fiction market, Rumbaugh says it's easier to win research funding to study duplicity and pathogenicity than it is to study the friendly 'symbiotic' and 'commensal' interactions in which one or both sides benefit.

As for Lyte, he too is pursuing the idea that an extensive and cordial dialogue is going on. He wants to examine whether bacteria use their signals to modulate complex host behaviours and functions, including learning and memory, and vice versa. "Bacteria are conversing with us, and we're conversing with them," says Lyte. The question now is how to record more of the conversation - and work out what is being said.

Asher Mullard is a freelance science writer based in London.

1. Nealson, K. H., Platt, T. \& Hasting, J. W. J. Bacteriol. 104, 313-322 (1970).

2. Lyte, M. \& Ernst, S. Life Sci. 50, 203-212 (1992)

3. Clarke, M. B., Hughes, D. T., Zhu, C., Boedeker, E. C. \& Sperandio, V. Proc. Natl Acad. Sci. USA 103, 10420-10425 (2006).

4. Iyer, L. M., Aravind, L., Coon, S. L., Klein, D. C. \& Koonin, E. V. Trends Genet. 20, 292-299 (2004)

5. Kravchenko, V. V. et al. Science 321, 259-263 (2008).

6. Hentzer, M. et al. Microbiology 148, 87-102 (2002).

7. Peterson, M. M. et al. Cell Host Microbe 4, 555-566 (2008).

8. Rasko, D. A et al. Science 321, 1078-1080 (2008). 